\title{
Relationships between post operative pain management and short term functional mobility in total knee arthroplasty patients with a femoral nerve catheter: A preliminary study
}

\author{
Catherine M Fetherston ${ }^{1 *}$, Sarah Ward ${ }^{2}$
}

\begin{abstract}
Background: Effective pain management following total knee arthroplasty (TKA) is fundamental in achieving positive rehabilitation outcomes. The purpose of our study was to investigate post operative pain management in relation to short term functional mobility in an intervention group receiving concomitant use of an IV narcotic PCA and a continuous infusion of local anaesthetic via a femoral nerve catheter (CFNC), compared to a group receiving narcotic PCA alone. This was a preliminary study conducted to establish an appropriate design for a larger investigative study.
\end{abstract}

Methods: A prospective design was used to measure the effect of a CFNC on post operative pain management and functional mobility prior to hospital discharge. The amount of fentanyl used, pain and nausea scores, timed up and go (TUG) tests and active range of knee movement (AROM) were used to compare a CFNC and supplemental narcotic patient controlled analgesia (PCA) group $(n=27)$ with a PCA only group $(n=25)$.

Results: The CFNC group used significantly less fentanyl than the PCA only group $(p<.001)$ but there was no significant difference in TUG times between the two groups. There was however a significantly lower AROM reported for both extension $(p<.04)$ and flexion $(p<.006$, ) in the FNC group. Women had significantly slower TUG times $(p<.005$,$) and there were moderate to strong positive correlations between post operative TUG times$ and the preoperative TUG time $\left(r_{s}=.505 p<.001\right)$, the time since oral analgesia $\left(r_{s}=.529 p<.014\right)$, and pain scores $\left(r_{\mathrm{s}}=.328, \mathrm{p}=.034\right)$

Conclusions: In this small preliminary study improved TUG performance at Day 4 post op was not influenced by the use of a CFNC but was positively correlated with male gender, preoperative performance, time elapsed since last oral analgesia and pain score. However AROM was decreased in the CFNC group suggesting further research on the relationship between CFNCs, local anaesthetic concentration and quadriceps strength should be incorporated in the follow up study's design.

\section{Background}

Effective pain management following total knee arthroplasty (TKA) is generally believed to be fundamental in achieving positive rehabilitation outcomes. It has been shown to be important for early physiotherapy and increased mobility and has been identified as an

\footnotetext{
* Correspondence: C.Fetherston@murdoch.edu.au

${ }^{1}$ School of Nursing and Midwifery, Murdoch University, Education Drive, Mandurah 6210, Western Australia

Full list of author information is available at the end of the article
}

influential factor in successful rehabilitation and reduced length of hospital stay [1,2]. However, a recent Danish study challenges this belief with their finding that pain has limited impact on functional recovery beyond the first post-operative day [3].

There are a range of methods that have been found to result in early effective postoperative pain control in patients undergoing TKA. These include intravenous (IV) patient controlled analgesia (PCA), intrathecal and epidural analgesia, lumbar plexus blockade, periarticular 
injection of local anaesthetics, single injection and continuous femoral nerve blockade, oral and intramuscular (IMI) narcotic and non narcotic analgesics [4]. Although IV narcotic PCA has been shown to be more effective than IMI and oral narcotic analgesia, and has the advantage of decreasing wait time for dose administration and dependency on nursing staff, it has been associated with significant adverse effects such as hypotension, confusion, pruritis, nausea and vomiting [5], all of which may potentially interfere with early postoperative mobility. Alternatively, the concomitant use of femoral nerve catheters (FNC) has been found to be associated with less PCA use, lower pain scores, and a shortened length of hospital stay [6-9] and as a result is becoming more appealing as a form of post operative analgesia. This popularity may not be justified in regard to continuous femoral nerve catheters (CFNC) as a review by the PROSPECT group (2008) concluded that although a femoral nerve block (FNB) was recommended based on evidence of reduced pain scores and supplemental analgesia, benefits of a continuous infusion compared to a single injection FNB may not be sufficient to justify the placement of catheters on a routine basis [10]. Further to this, a recent study has also found that extending an overnight CFNC to four days did not result in any increase in health related quality of life at either seven days or 12 months despite an increase in passive knee flexion during the infusion period $[9,11]$. Additionally, evidence in relation to the possible effects of continuous femoral nerve infusion on short term functional recovery is mixed. Two studies, one a retrospective pilot study [7] and the other a prospective randomised study [12] reported improved outcomes in the CFNC group in regard to increased flexion $[7,12]$ decreased mobility assistance requirements [7] and decreased length of hospital stay [7]. Whereas a further two randomised controlled studies $[13,14]$ reported no difference in either maximal knee flexion on post operative day (POD) 1 and 2 [13] or at discharge [14], or in the 2 min walk test conducted on PODs 1 to 3 [13]. There was also no difference in either time to first ambulation or length of hospital stay [14].

The purpose of our study was to investigate post operative pain management in relation to short term functional mobility in an intervention group receiving concomitant use of an IV narcotic PCA and a continuous infusion of local anaesthetic via a FNC, compared to a group receiving narcotic PCA alone. This was a preliminary study conducted to establish an appropriate design for a larger investigative study.

The research questions for this preliminary study were:

1. Is postoperative pain management improved in TKA patients who have a CFNC in addition to an intravenous narcotic PCA compared to patients whose pain is managed with narcotic PCA alone?

2 Is there a difference in expected and experienced levels of pain between the two comparison groups?

3. Is functional mobility at discharge improved in patients whose post operative analgesia is managed with concomitant use of an intravenous narcotic PCA and CFNC compared to those patients managed with narcotic PCA alone?

Outcome measures used in this preliminary study included patient's perceived pain, narcotic usage, and short term functional mobility. Functional mobility outcomes were measured using active range of knee movement (AROM) and a timed up and go test (TUG), prior to discharge.

\section{Methods \\ Design}

A quasi-experimental design was used where patients at a regional acute care hospital in Western Australia, who were undergoing a primary TKA, were followed prospectively to the fourth day (Day 4) after their surgery. University and hospital ethics committees' approvals were obtained prior to sequential sampling of those patients booked for a primary Press-fit TKA under the one orthopaedic surgeon. Recruitment of participants was undertaken between June and November 2008 and 64 patients were invited to take part in the study pre-operatively, either at the pre-admission clinic or on the surgical ward at the time of admission. Fifty seven patients consented to participate however five withdrew from the study postoperatively. This resulted in a final sample number of 52, of which $51.9 \%$ percent $(n=27)$ were managed by anaesthetist 1 , who used a continuous infusion of ropivacaine $0.2 \%$ via femoral nerve catheter in conjunction with supplemental IV fentanyl PCA. The remainder of the sample which constituted the PCA only group was managed by anaesthetist 2 who used only, either fentanyl PCA for $40.4 \%(n=21)$ or morphine PCA for $7.7 \%(n=4)$ of the group. As Anaesthetists 1 and 2 operated pre-admission clinics on alternate weeks, allocation of study participants to either the CFNC and supplemental PCA group or the PCA only group was not randomised, but occurred sequentially according to the order and timing of when booking documentation was received at the clinic.

In Anaesthetist 1's group a FNC was inserted post induction of anaesthesia, and prior to incision, via the guidance of a nerve stimulator technique. A loading dose of $20 \mathrm{ml} 0.75 \%$ ropivacaine and $20 \mathrm{ml} 2 \%$ lignocaine was administered in the recovery room prior to commencement of continuous infusion of $0.2 \%$ ropivacaine. Intravenous PCA for both groups was inserted in theatre and commenced either in recovery or on return to the ward depending on patient demand for analgesia. 
A physiotherapist assisted all post operative patients to stand out of bed on Day 1, the day following surgery, and mobilisation rehabilitation began on Day 2 and was supervised twice a day. Continuous Passive Movement (CPM) was commenced on the evening of Day 1 and was then supervised twice a day until discharge. Angle of flexion for CPM was aimed at achieving $30^{\circ}$ on Day $1,45^{\circ}$ on Day $2,60^{\circ}$ on Day 3 and $90^{\circ}$ on Day 4.

\section{Data collection/Instruments}

\section{Outcome measures for pain management}

Post operative perceived pain was recorded during routine post operative observations by nursing staff, who asked participants to self report their pain using a verbal rating scale (VRS) of $0-10$ where $0=$ no pain and $10=$ the worst pain imagined. Additionally a short questionnaire containing Likert scale questions designed to describe the expectations and experience of the participant's post operative pain was administered to participants on Day 4 post operatively.

\section{Outcome measures for functional mobility}

TUG tests and active range of knee movements (AROM) were both measured preoperatively (TUG1), at the time of recruitment, and then again post operatively on Day 4 (TUG2). The TUG2 s were measured either on the ward or in the physiotherapy department prior to the morning rehabilitation session. The TUG test, first described by Podsiadlo and Richardson [15] is a simple indicator of an older adult's functional mobility. It involves timing how long it takes the participant to stand up from a chair (seat height approximately $46 \mathrm{~cm}$ and arm height approximately $65 \mathrm{~cm}$ from the ground), walk 3 metres, turn and walk back to the chair and sit down. Results for TUG tests are categorised as worse than average if they exceed 9.0 secs for 60-69 years, 10.2 secs for 70-79 years and 12.7 secs for 80 to 99 years [16]. TUG tests were undertaken pre and postoperatively by the researchers or research assistant.

Preoperatively participants used a gait aid if this was how they normally mobilised. Crutches were used by $7.7 \%(\mathrm{n}=4)$ and a Zimmer frame by $3.8 \%(\mathrm{n}=2)$. Postoperatively elbow crutches were encouraged as the aid of choice although $25 \%(n=13)$ used a Zimmer frame as they had not yet developed confidence using crutches. Reliability and validity have been established previously $[15,17,18]$ and the inter-rater reliability for TUG test timing between researchers in this study was measured using pairwise correlation $(\mathrm{r}=1.0, \mathrm{p}<.001, \mathrm{n}=10)$. Range of knee movement was measured by one of the three treating physiotherapists using a universal goniometer. Intra and inter-rater reliability of the goniometer has been established previously using Pearson's intra-class correlation $\left(\operatorname{ICC}_{(1.1)}=0.99,0.90\right)$ [19].

\section{Demographic variables}

Demographic data and data on daily CPM, pain and nausea scores and type and doses of analgesia, local anaesthetic and anti-emetics received were collected from the patient's medical record, in addition to the number of PCA attempts by the patient versus the number of PCA delivered doses. As a wide range of antiemetics were prescribed by the two anaesthetists managing each group, nausea score was used as an outcome measure of nausea and vomiting in preference to amount of antiemetic used. Postoperative vomiting and nausea was classified as either: none (0), mild - intermittent nausea (1), moderate - dry retching (2) or, severe vomiting (3).

\section{Data Analysis}

Statistical analysis was conducted using Statistical Package for the Social Sciences (SPSS $17^{\circledR}$ ) for Windows (2008) [20]. Descriptive statistics were reported as mean and standard deviation (SD) or median and interquartile range (IQR) according to normality. As data were mixed in regard to normality a Mann Whitney $U$ test was used to compare data from the FNC and PCA only groups. Effects size were calculated as recommended by ClarkCarter [21] and classified by Cohen's [22] conventions where an $r=.1$ can be considered a small effect size, $r$ $=.3$ a medium effect and $r=.5$ a large effect. Correlational analyses were conducted using Spearman's Rho $\left(\mathrm{r}_{\mathrm{s}}\right)$ for ordinal and non-normal scale data . $P$ values less than 0.5 were considered significant for all data.

\section{Results}

\section{Demographic data}

The sample consisted of $44.2 \%(\mathrm{n}=23)$ male and $55.8 \%$ ( $\mathrm{n}=29$ ) female patients with more females than males in the CFNC group ( $\mathrm{n}=17$ and 10 respectively), than in the PCA only group ( $n=12$ and 13 respectively). Age was similar for both groups within the sample with a mean of $70 \pm 8.6$ years for the CFNC group and $70 \pm$ 7.3 years for the PCA only group, as was body mass index, $(29 \pm 5.5$ for the CFNC group and $30 \pm 3.9$ for the PCA only group). A right TKA was performed for $61.5 \%$ ( $n=32$ ) of participants, and a left TKA for $38.5 \%$ ( $\mathrm{n}=20$ ) with similar representation in the CFNC group (left $=10$, right $=17$ ) and the PCA only group (left = 10 , right $=15$ ). Mean degrees of flexion reached using CPM for each post operative day was not significantly different for either group (Table 1) and there was no significant difference in average length of stay which was $6.0 \pm 1.46$ nights for the CFNC group and $6.0 \pm$ 0.68 nights for the PCA only group. Patients in the CFNC group received a mean total dose of $354 \pm 71 \mathrm{~mL}$ of ropivacaine $0.2 \%$ over a mean duration of $36 \pm 5.8$ hours at an average hourly dose of $10 \pm 2.4 \mathrm{~mL}$. 
Table 1 Comparison of post operative flexion (mean \pm SD) attained using CPM for the FNC and PCA only groups

\begin{tabular}{lll}
\hline Day post op & FNC and PCA group & PCA only \\
\hline Day 1 Flexion (degrees) & $30.3 \pm 7.5$ & $29.4 \pm 7.7$ \\
Day 2 Flexion (degrees) & $43.8 \pm 10.4$ & $46.3 \pm 7.1$ \\
Day 3 Flexion (degrees) & $66.2 \pm 6.9$ & $67.1 \pm 7.8$ \\
Day 4 Flexion (degrees) & $83.3 \pm 7.3$ & $80.2 \pm 6.8$ \\
\hline
\end{tabular}

\section{Narcotic analgesia and pain scores}

There was no significant difference in the duration of PCA in the CFNC and PCA only groups (Table 2) and the amount of intravenous fentanyl used by both groups was compared. There was significantly more total and daily fentanyl used in the PCA only group compared to the CFNC group. This was also true for fentanyl used/ BMI. There were also significantly more dose increases required on the day of surgery in the PCA only group (Table 2).

The percentage of pain scores above 3, nausea scores above 0 and the highest reported pain scores on Day 2 were higher in the PCA only group although the difference was not significant. However the highest reported pain scores on both Day 0 (day of surgery) and Day 1 (day after surgery) were significantly higher (Table 3).

\section{Pain expectations}

The pain experienced by patients was either more, or a lot more, than expected in $48.1 \%(n=25)$ of the sample however there was no significant difference $(U=266, \mathrm{z}$ $=-.219, \mathrm{p}<.826)$ in the degree of perceived pain in relation to expectations between the CFNC and the PCA only groups.

\section{Functional mobility}

There was no significant difference in the type of gait aid used for either the CFNC or the PCA only group either pre- or postoperatively or in the preoperative TUG scores which were $12.5 \pm 4.4$ secs for the FNC group and $12.3 \pm 3.7$ for the PCA only group. Although the CFNC group had quicker postoperative TUG times the difference did not reach significance for this sample (Table 4) however there was significantly lower range of movement reported for both extension and flexion for the FNC group. There was no significant gender influence on AROM for either group (Table 5).

In the sample as a whole, men had quicker preoperative $(U=212.5, \mathrm{z}=-1.91, \mathrm{p}<.056$, medium effect size, $\mathrm{r}=.30)$ and postoperative TUG times $(U=123$, $\mathrm{z}=-2.78, \mathrm{p}<.005$, medium effect size, $\mathrm{r}=.42)$ than the women (Table 5). Men in the FNC group were also significantly quicker $(U=28, \mathrm{z}=-2.04, \mathrm{p}<.042$, small effect size, $r=.28$ ) and approached significance for the PCA only group $(U=31, \mathrm{z}=-1.91, \mathrm{p}<.056$, small effect size, $r=.26)$.

There were moderate to strong positive correlations between postoperative TUG (TUG2) scores and preoperative TUG (TUG1) scores $\left(\mathrm{r}_{\mathrm{s}}=.505 \mathrm{p}<.001\right)$ and the pain scores measured prior to TUG2 $\left(\mathrm{r}_{\mathrm{s}}=.328\right.$, $\mathrm{p}=.034)$, i.e. the faster the pre-op TUG time the better the patients performed post operatively; and the higher their pain score just prior to undertaking TUG2 the slower they performed. Additionally, the time lag since last pain medication prior to performing TUG2 was significantly higher for the FNC group (Table 4). This is reflected in a strong positive correlation $\left(\mathrm{r}_{\mathrm{s}}=.529\right.$ $\mathrm{p}<.014$ ) in the FNC group between time since last medication and TUG2 scores and a strong negative

Table 2 Comparison of fentanyl PCA data for patients with and without a FNC

\begin{tabular}{|c|c|c|c|}
\hline & FNC group $(n=27)$ & PCA only group $(n=21)$ & Statistical significance \\
\hline PCA duration (hours) & *37.7 \pm 2.8 & $39.6 \pm 4.4$ & NS \\
\hline Total fentanyl used $(\mu \mathrm{g})$ Day 0 & $450(220,73)$ & $820(609,1165)$ & $\begin{array}{l}\mathrm{p}<.001 \\
\text { Effect size medium, } \mathrm{r}=.48\end{array}$ \\
\hline Total fentanyl used $(\mu \mathrm{g})$ Day 1 & $1120(460,1560)$ & $1388(1080,2329)$ & $\begin{array}{l}p<.067 \\
\text { Effect size small, } r=.23\end{array}$ \\
\hline Total fentanyl used $(\mu \mathrm{g})$ Day 2 & $180(120,295)$ & $237(188,496)$ & $\begin{array}{l}p<.03 \\
\text { Effect size medium, } r=.31\end{array}$ \\
\hline Total fentanyl $(\mu \mathrm{g})$ used & $1860(800,2556)$ & $2820(2356,4238)$ & $\begin{array}{l}p<.001 \\
\text { Effect size large, } r=.52\end{array}$ \\
\hline Fentanyl used $(\mu \mathrm{g}) /$ hour & $51.8(22.8,66.8)$ & $89(61,104)$ & $\begin{array}{l}p<.001 \\
\text { Effect size large, } r=.52\end{array}$ \\
\hline Fentanyl dose $(\mu \mathrm{g}) / \mathrm{BMI}$ & $53.6(31.6,93.8)$ & $101(71,129)$ & $\begin{array}{l}p<.002 \\
\text { Effect size medium, } r=.45\end{array}$ \\
\hline Mean number of dose increases required on Day 0 & $0(0,1)$ & $1(0,1)$ & $\begin{array}{l}p<.016 \\
\text { Effect size medium, } r=.35\end{array}$ \\
\hline \% successful PCA attempts (Day 0) & $75(49,89)$ & $75.3(58.3,91.8)$ & NS \\
\hline$\%$ successful PCA attempts (Day 1) & $81.3(62.8,92.5)$ & $83.6(63.2,89.4)$ & NS \\
\hline
\end{tabular}

*Mean \pm SD, or median (IQR: 25,75$)$, reported according to normality 
Table 3 Comparison of post operative pain and nausea and vomiting scores

\begin{tabular}{llll}
\hline Post operative pain and nausea scores & FNC group $(\mathbf{n}=\mathbf{2 7})$ & PCA only group $(\mathbf{n}=\mathbf{2 1})$ & Statistical significance \\
\hline Average pain score (VRS) & $3.8(2.6,4.6)$ & $3.4(2.7,4.8)$ & NS \\
\% pain scores above 3 & $45.3 \pm 24.3^{*}$ & $48.5 \pm 25.9$ & NS \\
Day 0 highest reported pain score & $7.2 \pm 1.7$ & $8.36 \pm 1.7$ & $\mathrm{p}<.017$ Effect size medium, $r=.35$ \\
Day 1 highest reported pain score & $6(6,7)$ & $8(6,9)$ & $\mathrm{p}<.034$ Effect size medium, $r=.31$ \\
Day 2 highest reported pain score & $3.6 \pm 2.1$ & $4.3 \pm 2.8$ & NS \\
\% PONV scores above 0 ** & $3.3(3,12.5)$ & $6.4(0,14)$ & NS \\
\hline
\end{tabular}

*Mean \pm SD, or median (IQR: 25,75), reported according to normality.

**Postoperative nausea and vomiting (PONV) was classified as either: none (0), mild - intermittent nausea (1), moderate - dry retching (2) or, severe - vomiting (3).

correlation $\left(\mathrm{r}_{\mathrm{s}}=-.505 \mathrm{p}<.023\right)$ for the no FNC group (i.e. the lower the time lag between analgesia and performing TUG2 the better they did). However there was no such correlation between range of movement and time since last pain medication for either group (FNC group, Extension: $\mathrm{r}_{\mathrm{s}}=-.01 \mathrm{p}=.969$, Flexion: $\mathrm{r}_{\mathrm{s}}=.098$, $\mathrm{p}=.69$; the no FNC group, Flexion: $\mathrm{r}_{\mathrm{s}}=-.089 \mathrm{p}=.745$, Extension: $\mathrm{r}_{\mathrm{s}}=-.084, \mathrm{p}=.749$ ).

\section{Discussion}

Femoral nerve catheters are now used more frequently following TKA due to their improved efficacy in relation to lower pain scores, and an associated decrease in narcotic use post operatively. The benefits afforded by improved pain management related to the use of CFNCs, may also extend to benefits in short term functional recovery $[7-9,23]$ and this study has sought to contribute further knowledge in this area by investigating the relationship between the use of a CFNC and patient recovery in terms of their pain management, and functional mobility prior to discharge from hospital. Both the CFNC and the PCA only groups compared in this study had similar characteristics in regard to age, body mass index, operation site, length of hospital stay and whether a gait aid was used pre-operatively. There were also no significant differences in either the type of gait aid used postoperatively or in flexion attained when performing CPM exercises, whilst in hospital. Intravenous narcotic PCA was available to patients in both groups for a similar duration and both groups appeared to have a similar understanding of how to use PCA, as evidenced by comparable percentages of successful PCA dose attempts delivered. However there was a higher representation of women in the CFNC group than in the PCA only group.

This study's results have supported previous findings that patients with a CFNC use less supplemental narcotic analgesia during the postoperative period $[6,24,25]$. Having a CFNC in situ had a significantly large effect on the total fentanyl used with lower doses used, not only on the day of operation, but also on the first and second days postoperatively. The amount of fentanyl used also remained significantly lower when BMI was taken into account (Table 2). In general, patients in both groups appeared to manage their pain appropriately with an average pain score below 4 for both groups, however the highest pain score reported by patients was significantly higher (medium effect size) in the PCA only group on both the day of, and the day after, the operation. Although nausea scores were higher in the PCA only group, the difference did not reach significance in this sample and this is comparable with findings from previous studies $[6,24,26]$.

Short term functional mobility was measured using a TUG test and AROM on Day 4 following surgery, which was the day prior to assessment for hospital discharge on the clinical pathway. In a study that examined physical performance measures after TKA [18] the TUG

Table 4 Comparison of Day 4 Range of Movement, TUG and pain scores prior to TUG, for patients with and without a FNC

\begin{tabular}{|c|c|c|c|}
\hline Functional mobility, Day 4 post operatively & FNC $(n=27)$ & PCA only $(n=25)$ & Statistical significance \\
\hline AROM Flexion Day 4 (degrees) & $70.8 \pm 17.3^{*}$ & $82.6 \pm 15.8$ & $\begin{array}{l}p<.006 \\
\text { Effect size medium, } r=.38\end{array}$ \\
\hline AROM Extension Day 4 (degrees) & $-11.9 \pm 7.0$ & $-8.38 \pm 4.4$ & $\begin{array}{l}p<.040 \\
\text { Effect size small, } r=.29\end{array}$ \\
\hline TUG test post op (secs) & $45.23(31,67)$ & $58.0(39.5,71)$ & NS (outliers removed) \\
\hline Pain score at TUG test & $4.69 \pm 2.3$ & $3.67 \pm 2.5$ & NS \\
\hline Time elapsed since last analgesia at TUG test (hours) & *3.6 \pm 1.6 & $2.0 \pm 1.1$ & $\begin{array}{l}p<.003 \\
\text { Effect size medium } r=.42\end{array}$ \\
\hline
\end{tabular}

${ }^{*}$ Mean \pm SD, or median (IQR: 25,75$)$, reported according to normality. 
Table 5 Median (IQR) pre and post operative TUG test results (secs) and mean (SD) AROM (degrees) according to gender

\begin{tabular}{lllll}
\hline & & FNC group & \multicolumn{2}{c}{ PCA only group } \\
\hline & Male & Female & Male & Female \\
\hline Pre-op TUG (secs) & $9.9(9,11.2)$ & $13(10,14.1)$ & $10.3(9.4,15.6)$ & $11.5(9.6,16.1)$ \\
Post-op TUG 2 (secs) & $34(30.9,47.3)$ & $60.7(44.6,97.5)$ & $46.2(34.5,64.8)$ & $68.5(50,79.6)$ \\
Post-op Flexion & $* 72.4 \pm 11.9$ & $71.5 \pm 20.3$ & $83.1 \pm 15.3$ & $82.1 \pm 16.9$ \\
Post-op Extension & $-11.6 \pm 7.9$ & $-12.2 \pm 6.5$ & $-8.5 \pm 4.2$ & $-8.27 \pm 4.7$ \\
\hline
\end{tabular}

*Mean \pm SD, or median (IQR: 25,75), reported according to normality.

test was found to be useful in the early recovery period between one and nine to ten weeks postoperatively, by which time the test had reached a ceiling effect where most patients had met the 10 second criteria for being functionally independent [15]. Preoperative TUG results in our preliminary study indicate a degree of disability prior to surgery with the sample averaging a time of 12.4 seconds. This was greater for women, who had slower TUG times and supports previous research that found gender differences exist, with women reporting greater disability at the time of arthroplasty and lower self-reported function $[18,27,28]$. This may be explained by a study that found that women undergo arthroplasty at a more advanced disease state than men and also, as a result, had reduced muscle activation and increased atrophy preoperatively [27]. This level of disability was also reflected for women in our study's post operative TUG scores with women again having significantly slower times than men. It should also be noted that the researchers subjectively observed the men to be very competitive when completing their TUG tests, often asking the researchers how they compared to others and then "swapping results" on return to their shared rooms. Despite the gender influence, and the higher proportion of women in the FNC group, median TUG times were quicker for the FNC group (45 secs) than the PCA only group (58 secs), although the difference was not significant for this sample.

In comparison, the AROM was significantly lower in the CFNC group than in the PCA only group. Support for this finding is mixed. These results are not supported by previous findings from studies by Kadic et al. [12] and DeRuyter et al. [7] who found increased flexion on Days 3 to 6, and at Day 1 and discharge, respectively, in patients with a CFNC in situ for 48 hours. However a study by Carli et al. [13] using only a slightly lower dose regimen of $8 \mathrm{ml} / \mathrm{h}$, compared to the $10 \mathrm{ml} / \mathrm{h}$ of $0.2 \%$ ropivacaine in this study, found no significant difference in knee flexion on Days 1 and 2. The results in our study were not explained by the significant difference in the time analgesia was administered prior to testing, or by gender differences in each group. Most likely they can be explained by studies that found that quadriceps strength is the strongest predictor of functional performance $[29,30]$. This is a matter for concern in regard to short term functional mobility considering the recent published caution that femoral nerve blockade may result in prolonged quadriceps weakness and an increased risk of falls [31]. The variance in our results compared to the short term improvements seen in the study by Kadic et al. [12] may be related to a reduced dose of ropivacaine received by participants in their study. They described a dose of between 5 and $10 \mathrm{ml} / \mathrm{h}$ of $0.2 \%$ ropivacaine for the first $48 \mathrm{~h}$ (as opposed to an average dose of $10 \mathrm{ml} / \mathrm{h}$ in this study) although there were no details of the mean hourly or total dose received by participants making comparisons between the studies difficult. However recent data on the minimum local anaesthetic concentration (MLAC) showed that the minimum concentration at which patients did not require rescue analgesia using levobupivicaine was $0.024 \%$ for the femoral nerve and $0.014 \%$ for the sciatic nerve [32]. Even with this ultra-low concentration, which equates to a reduction in the commercial preparation's concentration of four to six fold, there was mild motor block manifested by an inability to dorsiflex, which prevented early mobilisation. This may indicate that the doses of ropivicane used in this study are related to the significantly lower AROM observed. However this may not be significant in terms of long term recovery as Kadic et al. also observed that short term improvements in knee flexion did not correlate with increased knee flexion and improved functional outcomes at three months.

The preoperative decline of quadriceps' strength and function has been shown to impact negatively on functional recovery $[33,34]$ and may also explain our findings of a strong positive correlation between slower preoperative TUG times and poorer postoperative TUG performance. However the correlations between slower TUG times and an increased time between analgesia and exercise, and a higher pain score, also reinforces the importance of timely effective analgesia prior to undertaking exercise in the post operative period prior to discharge. Our findings suggest that oral analgesia prior to exercise, once the femoral nerve catheter has been 
removed, is influential in improved functional mobility although this is at odds with recent findings from Denmark that pain has limited impact on functional recovery past the first postoperative day [3].

Positive impact on functional recovery may also be achieved through earlier surgical intervention to prevent severe disability prior to surgery [34-36]. However, TKA is often delayed by either wait lists or the requirement to decrease the need for future revision arthroplasty. In these cases improved preoperative function through the use of physical therapies has been shown to be effective in improving postoperative function [37].

\section{Study limitations and considerations for the follow-up study}

As a preliminary study this design was limited by the lack of probability sampling and small sample number. Subsequently there was a difference in gender representation with the increased proportion of females in the CFNC group being more representative of the general population, than in the PCA only group. This emphasises the importance of conducting a randomised design with an a priori power analysis in the follow-up study. Despite the absence of these design features in this preliminary study, the comparison groups were similar in their characteristics and the study was conducted prospectively with differences between groups identified as having medium to large effect sizes, so enabling important information for the conduct of the follow up study.

\section{Conclusions}

This study found that a CFNC was associated with important short term pain management benefits whilst insitu, in terms of significantly less supplemental narcotic use, and a lower number of narcotic PCA dose increases. Although the average pain score was not significantly different for the CFNC and PCA only groups the highest reported pain score was higher in the PCA only group on both the day of, and the day after the operation. The similar average pain scores reported in both groups is probably indicative that patients managed their pain appropriately with the assistance of rescue PCA, and that the CFNC patients required significantly less supplemental IV narcotic to achieve this. However the benefits observed for the CFNC whilst in situ, did not appear to extend to improved functional mobility after its removal. Three factors associated with reduced functional mobility on Day 4 post operatively were identified in this preliminary study. They were: gender, with women demonstrating slower TUG times; an increased time since oral analgesia was administered prior to mobilisation, which also accompanied a higher pain score; and a higher level of preoperative disability. This reinforces previous research that suggests there are several important factors that influence the short term post operative functional recovery after TKA. These include timely and effective analgesia prior to postoperative exercise and either timely surgery before marked muscle deactivation and atrophy occurs, or alternatively improving function prior to surgery using physical therapy. Of concern was the finding that AROM was significantly decreased in the CFNC group. This indicates that CFNC may be a variable of influence in the short term post-operative functional mobility of patients, especially in regard to concentrations of local anaesthetics used and its effects on quadriceps strength, and is therefore an important consideration in the follow up research.

\section{Abbreviations}

TKA: total knee arthroplasty; CFNF: continuous femoral nerve catheter; PCA: patient controlled analgesia; TUG test: timed up and go test; AROM: active range of motion; CPM: continuous passive movement; MLAC: minimum local anaesthetic concentration

\section{Acknowledgements}

We are grateful to the orthopaedic surgeon, Mr Michael Anderson and anaesthetists, Dr Murray Williams and Dr Ross Henderson, for their support and provision of patients for this study. Special thanks go to Research Assistant Jill Russell, physiotherapists Mark Kerns and Adam Beatty and the nursing staff for their support of the study. We would also like to acknowledge the very helpful advice provided by the reviewers.

\section{Author details}

${ }^{1}$ School of Nursing and Midwifery, Murdoch University, Education Drive, Mandurah 6210, Western Australia. ${ }^{2}$ Peel Health Campus, Lakes Road, Mandurah 6210, Western Australia.

\section{Authors' contributions}

CF conceived and designed the study, assisted with the data collection, performed the statistical analysis and prepared the manuscript

SW participated in the design of the study, data collection and manuscript preparation

\section{Competing interests}

The authors declare that they have no competing interests.

Received: 9 June 2010 Accepted: 7 February 2011

Published: 7 February 2011

\section{References}

1. Oldmeadow LB, McBurney H, Robertson VJ: Hospital stay and discharge outcomes after knee arthroplasty: Implications for physiotherapy practice. Aust J Physiother 2002, 48:117-121.

2. Capdevila $X$, Barthelet $Y$, Biboulet $P$, Ryckwaert $Y$, Rubenovitch J, d'Athis F: Effects of perioperative analgesia technique on the surgical outcome and duration rehabilitation after major knee surgery. Anesthesiology 1999, 91:8-15.

3. Holm B, Kristensen MT, Myhrmann L, Husted H, Andersen LO, Kristensen B, Kehlet $\mathrm{H}$ : The role of pain for early rehabilitation in fast track total knee arthroplasty. Dis Rehab 2010, 32:300-306.

4. Soever L, Mackay C: Best practices across the continuum of care for total joint replacement Ontario: Greater Toronto Area Rehabilitation Network; 2005.

5. Forst J, Wolff S, Thamm P: Pain therapy following joint replacement: A randomized study of patient-controlled analgesia versus conventional pain therapy. Arch Orthop Trauma Surg 1999, 119:267-270.

6. Singelyn FJ, Deyaert M, Joris D, Pendeville E, Gouverneur : Effects of intravenous patient controlled analgesia with morphine, continuous epidural analgesia, and continuous three-in-one block on postoperative pain and knee rehabilitation after unilateral total knee arthroplasty. Anesth Analg 1998, 87:88-92. 
7. De Ruyter ML, Brueilly KE, Harrison BA, Greengrass RA, Putzke JD, Borderson M: A pilot study on continuous femoral perineural catheter for analgesia after total knee arthroplasty. J Arthroplasty 2006, 21:1111-1117.

8. Iffeld BM, Gearen PF, Enneking FK, Berry LF, Spandoni EH, George SZ, Vandenbourne K: Total knee arthroplasty as an overnight-stay procedure using continuous femoral nerve blocks at home: a prospective feasibility study. Anaesth Analg 2006, 102:87-90.

9. Ilfeld BM, Le LT, Meyer RS, Mariano ER, Vandenbourne K, Duncan PW, Sessler DI, Enneking FK, Shuster JJ, Theriaque DW, Berry LF, Spandoni EH, Gearen PF: Ambulatory continuous femoral nerve blocks decrease time to discharge readiness after tricompartment total knee arthroplasty: a randomized, triple masked, placebo controlled study. Anesthesiology 2008, 108:703-13.

10. Fischer HBJ, Simanski CJP, Bonnet F, Camu EAM, Neugebauer N, Rawal N, Joshi GP, Schug SA, Kehlet H: A procedure specific systematic review and consensus recommendations for post operative analgesia following total knee arthroplasty. Anaesthesia 2008, 63:1105-23.

11. Ilfeld BM, Meyer RS, Le LT, Mariano ER, Williams BA, Vandenbourne $K$, Duncan PW, Sessler DI, Enneking FK, Shuster JJ, Maldonado RC, Gearen PF: Health-related quality of life after tricompartment knee arthroplasty with and without an extended-duration continuous femoral nerve block: $A$ prospective, 1-year follow-up of a randomized, triple masked, placebocontrolled study. Anaesth Analg 2009, 108:1320-25.

12. Kadic L, Boonstra MC, De Waal Malefijit MC, Lako SJ, van Egmond J, Driessen JJ: Continuous femoral nerve block after total knee arthroplasty. Acta Anaesthesio/ Scand 2009, 53:914-20.

13. Carli F, Clemente A, Asenjo JF, Kim DJ, Mistraletti G, Gomarasca M, Morabito A, Tanzer M: Analgesia and functional outcome after total knee arthroplasty: periarticular infiltration vs continuous femoral nerve block. Brit J Anaesth 2010, 105:185-95.

14. Seet E, Leong WL, Yeo ASN, Fook-Chong S: Effectiveness of 2-in-1 continuous femoral block of differing concentrations compared to patient controlled intravenous morphine for post total knee arthroplasty analgesia and knee rehabilitation. Anaesth Intensive Care 2006, 34(1):25-30.

15. Podsiadlo D, Richardson S: The timed "up and go": a test of basic functional mobility for frail elderly persons. J Am Geriatr Soc 1991 39:142-148.

16. Bohannon RW: Reference values for the timed up and go test: A descriptive meta-analysis. J Geriatr Phys Ther 2006, 29:64-68.

17. Freter SH, Fruchter N: Relationship between timed "up and go" and gait time in the elderly orthopaedic rehabilitation population. Clin Rehabil 2000, 14(1):96-101

18. Kennedy DM, Stratford P, Hanna SE, Wessel J, Gollish JD: Modeling early recovery of physical function following hip and knee arthroplasty. Musculoskeletal Disorders 2006, 7:100.

19. Watkins M, Reiddle D, Lamb R, Personius W: Reliability of goniometric measurement and visual estimates of knee range of motion obtained in a clinical setting. Phys Ther 1991, 71:90-96.

20. Statistical program for the social sciences SPSS Inc: III Chicago; 2008.

21. Clark-Carter D: Quantitative psychological research: A student's handbook New York: Psychology Press; 2004

22. Cohen J: A power primer. Psychol Bull 1988, 112:155-159.

23. Wang $H$, Boctor $B$, Verner J: The effect of single femoral nerve block on rehabilitation and length of hospital stay after total knee replacement. Reg Anaesth Pain Med 2002, 27:139-144.

24. Ganapathy S, Wasserman RA, Watson JT: Modified continuous femoral three in one block for postoperative pain after total knee arthroplasty. Anesth Analg 1999, 89:1197-1202.

25. Salinas FV, Liu SS, Mulroy MF: The effect of single-injection femoral nerve block versus continuous femoral nerve block after total knee arthroplasty on hospital leangth of stay and long term functional recovery within an established clinical pathway. Anesth Analg 2006, 102:1234-1239.

26. Serpell MG, Millar FA, Thompson MF: Comparison of lumbar plexus blockade versus conventional opiod analgesia after total knee replacement. Can J Anaesth 2004, 51:45, [Erratum appears in Can J Anaesth 2005; 52:119].

27. Petterson S, Bodenstab A, Snyder-Mackler L: Disease-specific gender differences among total knee arthroplasty candidates. J Bone Joint Surg 2007, 89A:2327-2333.
28. Katz JN, Wright EA, Guadagnoli E, Karlson EW, Cleary PD: Differences between men and women undergoing major orthopaedic surgery for degenerative arthritis. Arthritis Rheum 1994, 37:687-894.

29. Petterson SC, Mizner RL, Stevens JE, Raisis L, Bodenstab A, Newcomb W, Snyder-Mackler L: Improved function from progressive strengthening interventions after total knee arthroplasty: A randomized clinical trial with an embedded prospective cohort. Arthritis Rheum 2009, 61:174-183.

30. Mizner RL, Petterson AC, Snyder-Mackler L, et al: Quadriceps strength and the time course of functional recovery after total knee arthroplasty. $J$ Orthop Sports Physl Ther 2005, 35:424-436.

31. Kandasami M, Kinninmonth AWG, Sarungi M, Baines J, Scott N: Femora nerve blockade for total knee replacement - A word of caution. The Knee 2009, 16:98-100.

32. McLeod GA, Dale J, Robinson D, Checketts M, Columb MO, Luck J, Wigderowitz C, Rowley D: Determination of the $\mathrm{EC}_{50}$ of levobupivacaine for femoral and sciatic perineural infusion after total knee arthroplasty. Brit J Anaesth 2009, 102:528-33.

33. Mizner RL, Petterson SC, Stevens JE, Axe MJ, Snyder-Mackler L: Preoperative quadriceps strength predicts functional ability one year after total knee arthroplasty. J Rheumatol 2005, 32:1533-1539.

34. Fortin PR, Penrod JR, Clarke AE, St-Pierre Y, Joseph L, Belisle P, Liang MH, Ferland D, Phillips CB, Mahomed N, Tanzer M, Sledge C, Fossel AH, Katz JN: Timing of total joint replacement affects clinical outcomes among patients with osteoarthritis of the hip or knee. Arthritis Rheum 2002, 46:3327-3330.

35. Lingard EA, Katz JN, Wright EA, Slege CB: Predicting the outcome of total knee arthroplasty. J Bone Joint Surg Am 2004, 86:2179-2186.

36. Kennedy LG, Newman JH, Ackroyd CE, Dieppe PA: When should we do knee replacements? Knee 2003, 10:161-116.

37. Jones CA, Voaklander DC, Suarez-Almazor ME: Determinants of function after total knee arthroplasty. Phys Therap 2003, 83:696-706.

doi:10.1186/1749-799X-6-7

Cite this article as: Fetherston and Ward: Relationships between post operative pain management and short term functional mobility in total knee arthroplasty patients with a femoral nerve catheter: A preliminary study. Journal of Orthopaedic Surgery and Research 2011 6:7.

\section{Submit your next manuscript to BioMed Central and take full advantage of:}

- Convenient online submission

- Thorough peer review

- No space constraints or color figure charges

- Immediate publication on acceptance

- Inclusion in PubMed, CAS, Scopus and Google Scholar

- Research which is freely available for redistribution

Submit your manuscript at www.biomedcentral.com/submit
C Biomed Central 